\title{
A clinical study on maternal and fetal outcome in pregnancy with oligohydromnios
}

\section{Chetan Prakash Gupta*, Jaya Choudhary, Deepika Chahar, Sapna Kumari Yadav}

Department of Obstetrics and Gynecology, Mahatma Gandhi Medical College and Hospital, Jaipur, Rajasthan, India

Received: 15 September 2018

Accepted: 06 October 2018

\section{*Correspondence:}

Dr. Chetan Prakash Gupta,

E-mail: drchetangupta79@gmail.com

Copyright: (C) the author(s), publisher and licensee Medip Academy. This is an open-access article distributed under the terms of the Creative Commons Attribution Non-Commercial License, which permits unrestricted non-commercial use, distribution, and reproduction in any medium, provided the original work is properly cited.

\begin{abstract}
Background: Oligohydramnios is associated with various maternal and fetal complication. It's correlated fetal complications like FGR, increased risk of meconium aspiration syndrome, Birth asphyxia, low APGAR scores and increased congenital abnormalities in fetus. It's also associated with maternal morbidities in view of operative interventions for delivery. To study effect of oligohydramnios in mother in form of, operative delivery and progress of labour. To study effect of oligohydramnios in fetus in form of fetal compromise i.e. FGR, fetal distress, altered APGAR score, need for NICU admission. congenital anomaly and perinatal death.

Methods: 50 patients with $\geq 28$ weeks POG with oligohydramnios, confirmed by ultrasonographic measurement of AFI using four quadrant technique; were selected randomly after fulfilling inclusion and exclusion criteria.

Results: Incidence of oligohydramnios were more found in primigravida (56\%) in present study. Most common cause of oligohydramnios was idiopathic (62\%) followed by PIH (20\%). Most common cause of caesarean was fetal distress $(23 \%)$ either due to cord compression or FGR. oligohydramnios was related to higher rate of Fetal Growth restriction and NICU admission (24\%).

Conclusions: Oligohydramnios is very common encounter during pregnancy, Because of its frequent occurrence; it demands intensive fetal monitoring and systemic approach to antepartum and intrapartum fetal surveillance. There is increased risk of intrapartum complication, perinatal morbidity, perinatal mortality; thus, the rate of caesarean increasing day by day.one should always know the fine line between vaginal delivery and caesarean section; move ahead with best option without hampering fetal wellbeing and avoid unnecessary operative morbidity.
\end{abstract}

Keywords: Fetal outcome, Maternal outcome, Oligohydromnios, Pregnancy

\section{INTRODUCTION}

Amniotic fluid provides protected shield for the growing fetus, cushioning fetus against mechanical and biological injury, supplying nutrient and facilitating growth and movement of fetus. Early in the developmental period of fetus, it is enclosed by amnion and is surrounded by amniotic fluid which is similar to extracellular fluid and an indicator of fetal status that has made amniotic fluid volume assessment, an important part of antenatal fetal surveillance. The quantity of amniotic fluid changes according to period of gestation. Oligohydromnios refers to amniotic fluid volume that is less than expected for gestational age. It is typically diagnosed by ultrasound examination and may be described qualitatively (e.g., normal, reduced) or quantitatively (e.g., amniotic fluid index $[\mathrm{AFI}] \leq 5) .{ }^{1}$ Oligohydramnios occurs in about $1-5 \%$ of pregnancies at term. ${ }^{2}$ In pregnancies of more than 40 weeks of gestation, the incidence may be more than $12 \%$ as the amniotic fluid volume declines progressively after 41 weeks of gestation. ${ }^{3}$ Oligohydromnios results from medical or obstetrical complication related to maternal ,placental ,fetal causes and idiopathic. Both abnormal, increase and decrease in amniotic fluid volume have been associated with maternal as well as fetal morbidity and mortality. With advent of real time USG better 
identification can be done using AFI method described by phalen et al, where four quadrant technique is employed during TAS. ${ }^{1}$ Oligohydromnios is associated with congenital fetal anomalies, Uteroplacental insufficiency, Premature Rupture of membranes, postdatism, abruption placenta and hypertensive disorder in pregnancy. It is found to be associated with high incidence of maternal and fetal morbidity and mortality. During labour the predominant mechanical function of amniotic fluid is to provide an aquatic cushion for umbilical cord. Without this cushion, compression of the cord between the fetus and the uterine wall may occur during contractions or fetal movement, this cord compression causes fetal distress which are associated with low APGAR scores and acidosis at birth, meconium staining, caesarean section and operative vaginal delivery. Early detection of oligohydramnios and its management may help in reduction of perinatal morbidity and mortality one side and decreased rate of caesarean deliveries on the other side. The objectives of the present study was to observe the effect of oligohydramnios in maternal outcome in form of, operative delivery and progress of labour, and to study the effect of oligohydramnios in fetal outcome in form of fetal compromise i.e. FGR, fetal distress, altered APGAR score, Need for an NICU admission, congenital anomaly and perinatal death.

\section{METHODS}

This study was conducted in department of Obstetrics and Gynecology of Mahatma Gandhi Medical College and Hospital, Jaipur; between Nov 2017 to June 2018. 50 patients with $\geq 28$ weeks POG with oligohydramnios, confirmed by ultrasonographic measurement of AFI using four quadrant technique; were selected randomly after fulfilling inclusion and exclusion criteria.

\section{Inclusion criteria}

- $\quad$ Singleton pregnancy

- Pregnancy $\geq 28$ weeks POG with intact membrane

- $\quad$ USG proven AFI upto $5 \mathrm{cms}$.

\section{Exclusion criteria}

- Patient with Premature rupture of membrane

- Multiple pregnancy.

A detailed history and examination were done, oligohydromnios confirmed by AFI. Routine management in form of rest, left lateral position, oral and intravenous hydration and control of etiological factor was done if present. Fetal surveillance was done by USG, modified biophysical profile and Doppler. Decision of delivery by either induction or elective or emergency LSCS was done as per required. Some patients were already in labour and other allows going in spontaneous labour.

\section{RESULTS}

Out of 50 patients ,52\% of patients were in 20-25 years age group and $36 \%$ were in 26-30 age group and rest $8 \%$ and $4 \%$ in $30-35$ years, $<20$ years age group simultaneously. Thus, maximum Patients were in 20-30 years age Group. More number of sections was highest in 20-25 years age group, and lowest in $<20$ years age group.

Table 1: Age and maternal outcome (mode of delivery) in oligohydramnios.

\begin{tabular}{|llllll|}
\hline Age (Years) & Vaginal delivery & & LSCS & Total & Percentage \\
\hline$<20$ & Normal & Assisted & $1(50 \%)$ & 2 & 4 \\
\hline $20-25$ & $1(50 \%)$ & $1(3.84 \%)$ & $11(42.30 \%)$ & 26 & 52 \\
\hline $26-30$ & $14(53.84 \%)$ & & $9(50 \%)$ & 18 & 36 \\
\hline $30-35$ & $9(50 \%)$ & & $2(50 \%)$ & 4 & 8 \\
\hline Total & $2(50 \%)$ & $1(2 \%)$ & $23(46 \%)$ & 50 & 100 \\
\hline
\end{tabular}

Table 2: Parity and maternal outcome of labour in oligohydramnios.

\begin{tabular}{|c|c|c|c|c|c|}
\hline \multirow{2}{*}{ Parity } & \multicolumn{2}{|c|}{ Vaginal delivery } & \multirow{2}{*}{ LSCS } & \multirow{2}{*}{ Total } & \multirow{2}{*}{ Percentage } \\
\hline & Normal & Assisted & & & \\
\hline primigravida & $12(42.8 \%)$ & $1(3.57 \%)$ & $15(53.57 \%)$ & 28 & 56 \\
\hline multigravida & $14(63.63)$ & - & $8(36.36)$ & 22 & 44 \\
\hline Total & $26(52 \%)$ & $1(2 \%)$ & $23(46 \%)$ & & 100 \\
\hline
\end{tabular}

Incidence of oligohydromnios was highest in primigravida $(56 \%)$ followed by $44 \%$ in multigravida in present study. And operative morbidity was also highest in primigravida $(53.57 \%)$ followed by $36.36 \%$ in multigravida. Most common cause of oligohydromnios is idiopathic $(64 \%)$. Second commonest cause is 
Hypertensive disorder in pregnancy $(20 \%)$. operative morbidity is highest in hypertensive disorder in pregnancy $(60 \%)$.

Oligohydromnios was associated with post-dated pregnancy $(8 \%)$, fever $(4 \%)$, post term $(2 \%)$ and severe anemia $(2 \%)$.

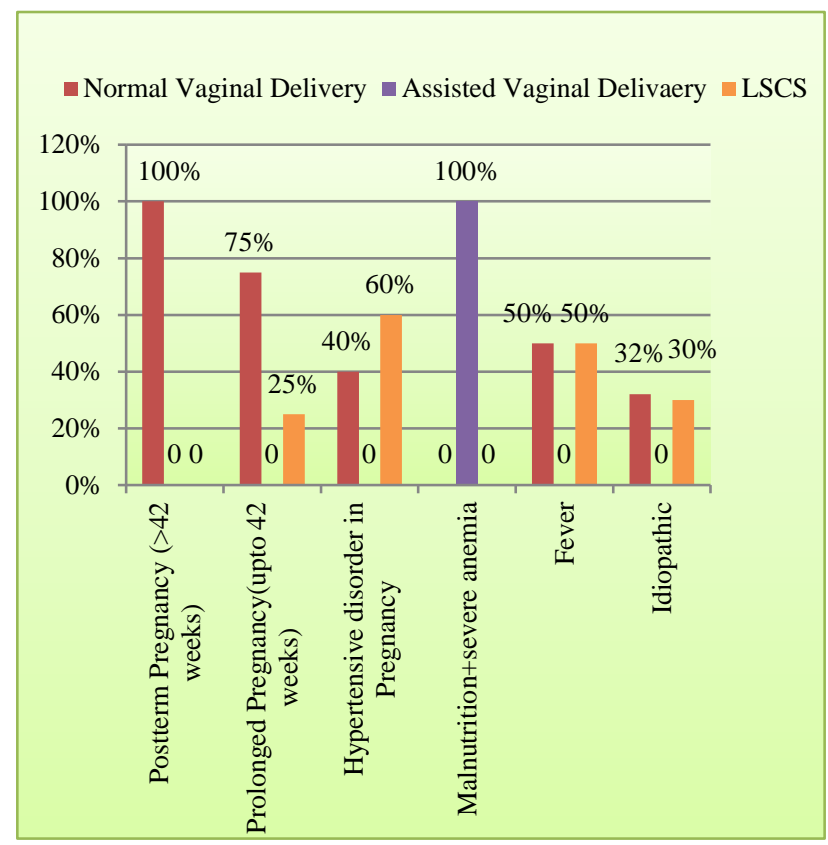

Figure 1: Associated condition and maternal outcome in oligohydromnios

Out of 50 patients, $38(76 \%)$ patients had reactive nonstress test in which $34.21 \%$ patients had LSCS.

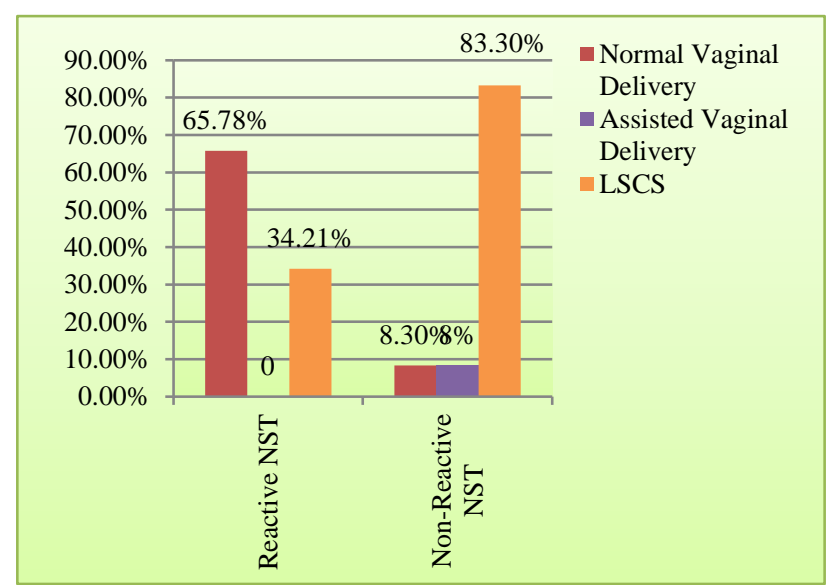

Figure 2: Non-stress test in oligohydromnios.

Operative intervention was significantly higher in NST non-reactive group (24\%) in which 83.3 patients had LSCS. incidence of normal vaginal delivery was $65.78 \%$ in NST reactive Patients.

All patients were undergone Doppler study.14\% patients were found to have placental insufficiency.
Out of them, Operative intervention was significantly higher $(85.71 \%)$ in patient with abnormal Doppler findings. $86 \%$ patient had normal Doppler study in which $39.53 \%$ patients underwent LSCS.

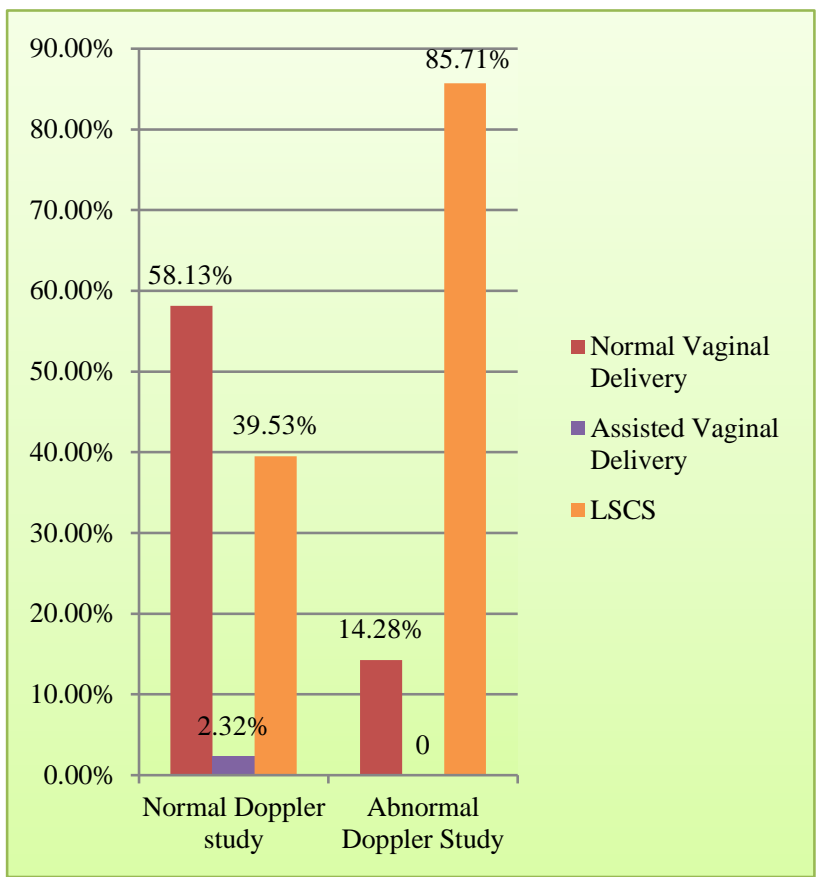

Figure 3: Doppler study in oligohydromnios

In present study most, common indication of LSCS was fetal distress (23\%). Oligohydromnios, FGR, Breech was indication of LSCS in $10 \%, 9 \%$ and $2 \%$ of patients simultaneously.

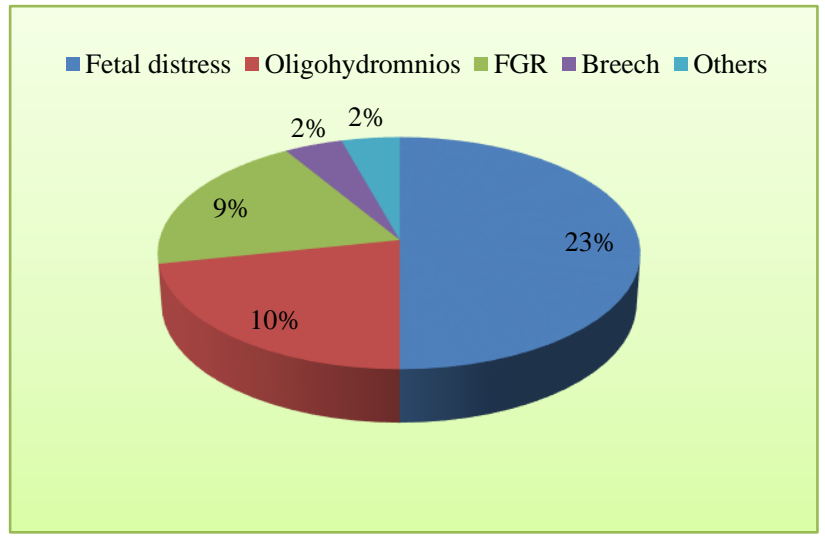

Figure 4: Indications of LSCS

In present study Fetal Growth restriction was present in form of $80 \%$ of AGA and $20 \%$ for SGA. APGAR score ( $<7$ in 1 and 5 minutes) was present in $17 \%$ of cases. NICU admission needed in $24 \%$ of new-born.

Congenital anomaly and perinatal death was present in $2 \%$ of cases each. 


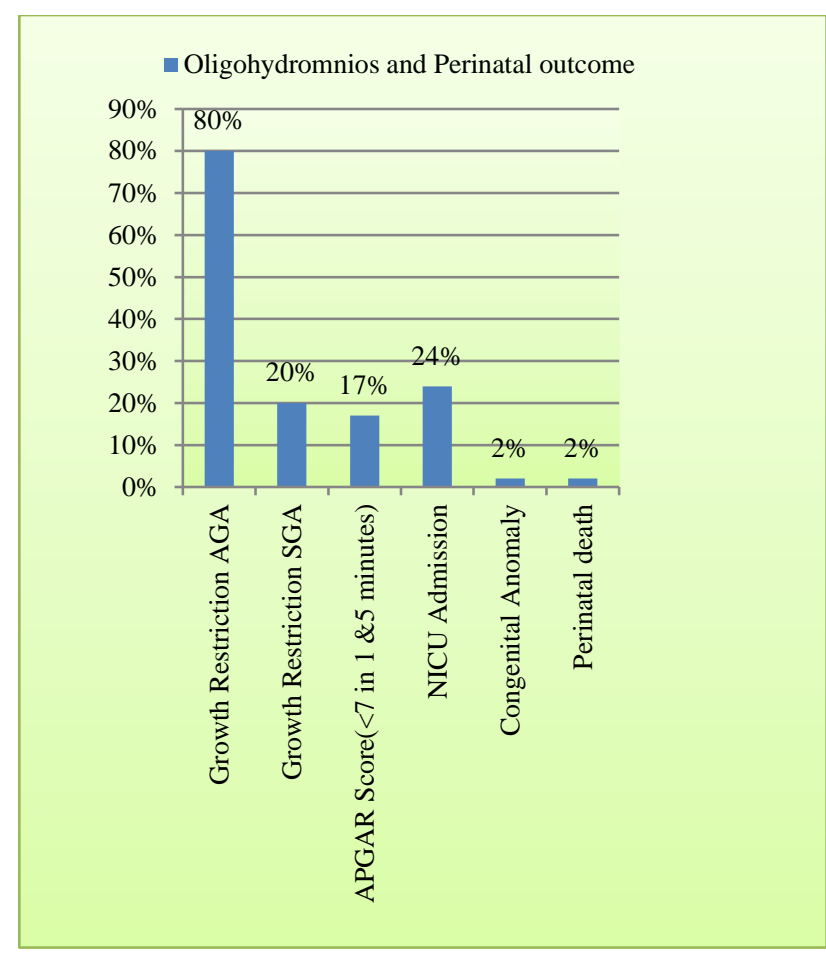

Figure 7: Oligohdromnios and perinatal outcome

\section{DISCUSSION}

In present study the incidence of oligohydromnios in primigravida is $56 \%$, which is comparable to study done by Donald et al, it was $60 \%$. It was $52 \%$ in study conducted by Jagatia $\mathrm{K}$ et al. ${ }^{4,5}$

Various studies represent different rate of LSCS in pregnant women with amniotic fluid index $<5 \mathrm{~cm}$. The LSCS was done in $46 \%$ of cases in present study which is compared with other studies as follow. study by Casey B et al, found that there was increased rate (32\%) of caesarean section in oligohydromnios cases. ${ }^{6}$ Golan et al [7],found that, the caesarean section was performed in $35.2 \%$ of cases of oligohydromnios. ${ }^{7}$ Bansal D et al,found that there was $46 \%$ of cases of oligohydromnios who undergone caesarean section. These studies are comparable to my study. ${ }^{8}$

Most common cause of oligohydromnios reported by Jagatia $\mathrm{K}$ et al. was idiopathic followed by hypertensive disorder in pregnancy which is comparable to my study. in present study association of hypertensive disorder is $20 \%$ which is comparable to study by Sriya et al, in which its incidence was $31 \% .5,98 \%$ cases had postdated pregnancy in present study. Marks and divon has reported oligohydromnios in $11.5 \%$ of 551 pregnancies at 41 weeks or greater. ${ }^{10} \mathrm{~A}$ study done by Bhat et al at Bharati Vidyapeeth Deemed University Medical College and Hospital at Sangli including 100 patients in third trimester of pregnancy with Oligohydramnios; found that the most common cause of oligohydramnios was idiopathic followed by PIH. ${ }^{11}$ In the study by Kumar et al $40 \%$ of patients had non-reactive NSTs, while in the study by Chandra et al it was $69.23 \%$ and in the study by Sriya et al. it was $41.55 \% .^{9,12,13}$ In present study it was $24 \%$. The operative morbidity is significantly higher in patients with altered Doppler study. In Weiss et al and Yound $\mathrm{HK}$ et al it was $71 \%$ and $69.7 \%$ respectively which was comparable to this study, in present study it was $83.3 \% .^{14,15}$

In present study, we had intrapartum complication in form of fetal distress (23\%). Which is comparable to study done by Casey et al (32\%), most common reason to perform caesarean was fetal distress which was either due to cord compression or FGR. ${ }^{6}$ Results are comparable with Casey et al study, which show significant higher proportion of LSCS due to fetal distress. There is high rate of operative delivery (instrumental + LSCS) in all the studies which are comparable to present study. Thus, oligohydramnios is associated with increased operative delivery and therefore, increased maternal morbidity.

In Julie Johnson et al [16] $92.6 \%$ babies were AGA and $7 \%$ were SGA. In Brain M Casey et al [6] $75.5 \%$ babies were AGA and 24\% SGA. In Philipson, EH et al babies were $60 \%$ AGA and $40 \%$ SGA. In Manning et al babies were $64 \%$ AGA and $36 \%$ SGA. ${ }^{17,18}$ In Sriya et al babies were $83.4 \%$ AGA and $16.6 \%$ SGA. This high percentage of SGA babies suggesting correlation of FGR with Oligohydramnios. $^{9}$ In Manning et al $15 \%$ babies had APGAR score $<7$ at 5 minutes. ${ }^{18}$ In Sriya et al, it was $38 \%$, in present study it was found to be $17 \%$. In Julie M Jhonson et al $20 \%$ babies had NICU admission. In Manning et al and Sriya et al, $43 \%$ and $88.88 \%$ respectively. ${ }^{9,16,18}$ In present study $24 \%$ babies required NICU admission. Golan et al show $6.3 \%$ perinatal death in deliveries of Oligohydramnios patients which is observed $2 \%$ in present study. ${ }^{7}$

\section{CONCLUSION}

Oligohydramnios is frequent occurrence and demands intensive fetal surveillance and proper antepartum and intrapartum care.

Oligohydramnios is a frequent finding in high risk pregnancies like FGR, hypertensive disorder in pregnancy, pregnancy beyond 40 weeks of gestation. AFI is a predictor of fetal tolerance in labour and decrease in AFI is associated with increased risk of abnormal fetal heart rate and meconium stained liquor. Due to intrapartum complications and high rate of caesarean section is rising, but decision between vaginal delivery and caesarean section should be well balanced so that unnecessary maternal morbidity prevented and on other side timely intervention can reduce perinatal morbidity and mortality.

\section{Funding: No funding sources}

Conflict of interest: None declared

Ethical approval: The study was approved by the Institutional Ethics Committee 


\section{REFERENCES}

1. Phelan JP, Smith CV, Broussard P, Small M. Amniotic fluid volume assessment with four quadrant technique at 36-42 weeks of gestation. J Reprod Med. 1987;32(7):540-54.

2. Moore IR. Clinical assessment of amniotic fluid. Clin Obstet Gynaecol. 1997;40(2):303-13.

3. Sherer DM, Langer O. Oligohydramnios: use and misuse in clinical management. Ultrasound Obstet Gynaecol. 2001;18(5):410-1.

4. Petrozella LN, Dashe JS, McIntire DD, Leveno KJ. Clinical Significance of Borderline Amniotic Fluid Index and Oligohydramnios in Preterm Pregnancy. Obstet Gynecol 2011;117(2 Pt 1):338-42

5. Jagatia K, Singh N, Patel S. Maternal and fetal outcome in oligohydramnios: A study of 100 cases. Int J Med Sci Public Health 2013; 10(40):724-7.

6. Casey BM, McIntire DD, Bloom SL, Lucas MJ, Santos R, Twickler DM, et al. Pregnancy outcomes after antepartum diagnosis of oligohydramnios at or beyond 34 weeks' gestation. Am J Obstet Gynecol. 2000;182(4):909-12.

7. Golan A, Lin G, Evron S, Arieli S, Niv D, David MP. Oligohydramnios: maternal complications and fetal outcome in 145 cases. Gynecol Obstet Investigat. 1994;37(2):91-5.

8. Bansal D, Deodhar P. A Clinical Study of Maternal and Perinatal Outcome in Oligohydramnios. J Res Med Den Sci. 2015;3(4):312-6

9. Sriya $R$, Singhai S. Perinatal outcome in patients with amniotic fluid index $<5 \mathrm{~cm}$. J Obstet Gynaecol India 2001;51:98-100.

10. Marks AD, Divon MY : Longitudinal study of the amniotic fluid index in post-dates pregnancy. Obstet Gynecol 1992;79(2):229-33

11. Bhat S, Kulkarni V. Study of effect of oligohydramnios on maternal and fetal outcome. Int J Med and Dent Sci. 2015;4(1):582-8.
12. Kumar P, Iyer S, Ramkumar V. Amniotic fluid index a new ultrasound assessment of amniotic fluid. $\mathbf{J}$ Obstet Gynaecol India. 1991;41(1):10-2.

13. Chandra P, Kaur SP, Hans DK, Kapila AK. The impact of amniotic fluid volume assessed intrapartum on perinatal outcome. Obstet Gynaecol. 2000;5(8):178-81

14. Hitschold $T$, Weiss E, Berle $P$, Muntefering $H$. Histologic placenta findings in prolonged pregnancy: correlation of placental retarded maturation, fetal outcome and Doppler sonographic findings in the umbilical artery. Z Geburtshilfe Perinatol 1989;193(1):42-6

15. Kwon JY, Kwon HS, Kim YH, Park YW. Abnormal Doppler Velocimetry is related to adverse perinatal outcome for borderline amniotic fluid index during third trimester. J Obstet Gynecol Res 2006:32(6):545-9.

16. Johnson JM, Chauhan SP, Ennen CS, Niederhauser A, Magann EF. A comparison of 3 criteria of oligohydramnios in identifying peripartum complications: a secondary analysis. Am J Obstet Gynecol 2007;197(2):207.e1-7

17. Philipson EH, Sokol RJ, Williams T. Oligohydramnios: Clinical Associations and Predictive Value for Intrauterine Growth Retardation. Am J Obstet Gynecol 1983;146(3):2718.

18. Manning FA, Hill LM, Platt LD. Qualitative amniotic fluid volume determination by ultrasound: Antepartum detection of intrauterine growth retardation. Am J Obstet Gynecol 1981;139(3):2548.

Cite this article as: Gupta $\mathrm{CP}$, Choudhary $\mathrm{J}$, Chahar D, Yadav SK. A clinical study on maternal and fetal outcome in pregnancy with oligohydromnios. Int $\mathrm{J}$ Reprod Contracept Obstet Gynecol 2018;7:4731-5. 